\title{
Fractal pharmacokinetics
}

\author{
Luis M. Pereira* \\ Harvard Medical School, Children's Hospital Boston, Boston, USA \\ (Received 23 February 2009; final version received 7 May 2009)
}

\begin{abstract}
Pharmacokinetics (PK) has been traditionally dealt with under the homogeneity assumption. However, biological systems are nowadays comprehensively understood as being inherently fractal. Specifically, the microenvironments where drug molecules interact with membrane interfaces, metabolic enzymes or pharmacological receptors, are unanimously recognized as unstirred, space-restricted, heterogeneous and geometrically fractal. Therefore, classical Fickean diffusion and the notion of the compartment as a homogeneous kinetic space must be revisited. Diffusion in fractal spaces has been studied for a long time making use of fractional calculus and expanding on the notion of dimension. Combining this new paradigm with the need to describe and explain experimental data results in defining time-dependent rate constants with a characteristic fractal exponent. Under the one-compartment simplification this strategy is straightforward. However, precisely due to the heterogeneity of the underlying biology, often at least a two-compartment model is required to address macroscopic data such as drug concentrations. This simple modelling step-up implies significant analytical and numerical complications. However, a few methods are available that make possible the original desideratum. In fact, exploring the full range of parametric possibilities and looking at different drugs and respective biological concentrations, it may be concluded that all PK modelling approaches are indeed particular cases of the fractal PK theory.
\end{abstract}

Keywords: fractional calculus; anomalous diffusion; fractal microenvironments; time-dependent rate constants

\section{Introduction}

Since the notion of 'compartment' was first introduced by Teorell [72] to describe the time course of drugs in biological systems, the homogeneity assumption has become almost dogmatic in pharmacokinetics (PK) $[61,75,80,83]$. Amenable to the differential calculus formalism, such a compartment, or homogeneous kinetic space, is really an abstraction that together with the law of mass action continues to be very useful in the context of compartmental analysis. It bears no anatomical or physiological connotation other than referring to the ensemble of all the tissues, organs or systems for which the probability of encountering a drug molecule at any given point is the same. This assumption builds upon another about Fickean diffusion for which the mean square displacement of a drug molecule in a homogeneous, or well-stirred, space is proportional to time $\left(\left\langle x^{2}(t)\right\rangle \propto t\right)$. However, phenomena referred to as anomalous diffusion have been known for a long time, even preceding Teorell's work. Richardson conjectured, although empirically, in 1926, that the diffusion coefficient in a turbulent medium depends on the scale unit of the measurement

\footnotetext{
*Email: luis.pereira@childrens.harvard.edu
} 
[62], defining the Richardson plot principle still used today. Later, unusual experimental observations started being reported as 'strange' kinetics [9] and, after Mandelbrot's seminal work [49], many reports followed, for example, on diffusion in dense objects [42], dynamics in polymeric networks [2,8], diffusion in porous and fractal media [55,59] and kinetics in viscoelastic media [48]. Ultimately, a generalized diffusion equation was proposed $[41,56]$ which provided a heuristic support for a more realistic characterization of systems with dimensional constraints, as biological microenvironments are indeed.

Kopelman's groundbreaking work on chemical kinetics [3,34,35] became the foundation for general fractal kinetics and PK just followed [33]. Particularly in the PK field, Macheras [44] and Savageau [66] were among the first to document real fractal PK applications and since then many other examples were published, particularly by Macheras' group, for modelling drug dissolution [20,36,38-40,47,77], absorption $[13,25,46]$, distribution [21,28-32,45], whole disposition [79], stochastic processes [16], Michaelis-Menten metabolism [50] and carrier-mediated transport [58].

However, most of the publications on fractal PK so far, are phenomenological or very specific in scope and only a few attempt to establish a theoretical quantitative methodology. Kotulski et al. [37] made use of stochastic relaxation theory in the context of power law time dependent rate constants for first- and second-order kinetics with bimolecular reactions. Later, Tsallis [73] used non-extensive entropy theory to derive nonlinear differential equations with fractional-order, but still a theoretical PK approach is missing. This work intends to systematize the background theory and provide a unified modelling framework for handling PK experimental data.

\section{Theory}

Although fractal kinetics is usually built up from the fractal geometry point of view, it has more to do with anomalous diffusion and fractional calculus than anything else. Homogeneity, in the sense of instantaneous mixing, is really not supported by nature, particularly in microenvironments, space restricted and heterogeneous, sometimes impacting on how whole systems behave at the macro-level. Namely, fixed rate constants mean the fraction of drug transferred from one compartment to another is fixed, independent of time and amount. This is true only under the homogeneity assumption.

Conversely, heterogeneous media may be conceived as low-dimension environments or fractal spaces, for which their dimension $d$ is fractional. As an example, one may think about a pathway along a simple line versus a whole surface admissible for movement, being obvious that the first has dimension equal to one, or one degree of freedom, while the latter has dimension two for two degrees of freedom (Figure 1(I),(II)). These are the Euclidean dimensions which assume only integer values. But imagining the path getting increasingly tortuous, as trying to 'fill' the surface, then its real length depends on the 'ruler' used to measure it (Figure 1(III)). As the smallest division of this 'ruler' gets smaller, more fine characteristics of the line are captured adding to the path length

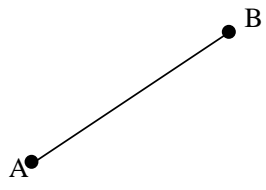

I

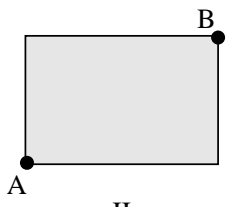

II

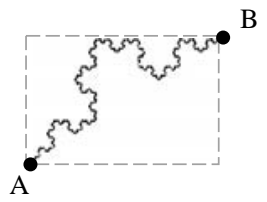

III

Figure 1. Examples of a line, a surface and something in between. 

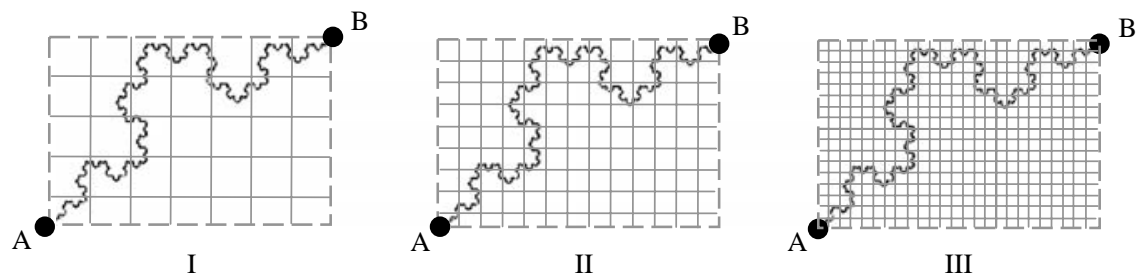

Figure 2. Illustration of the box-counting method to determine the dimension of a fractal object.

(Figure 2). On the limit, for an infinitely small particle, if the line had infinitely intricate details, its length would be infinite. In practice, moving particles have a finite size, and when a relationship is found between the measurements of the path and their unit size, it's usually of a $\log -\log$ type and corresponds to a constant real number defined as fractal dimension.

Perhaps the simplest way to identify and estimate the fractal dimension of an object is the so-called box-counting method. Basically, one defines different grids superimposed on a projection of the object (Figure 1(III)), keeping track of the 'unit size' and the number of boxes required to cover it (Figure 2(I)-(III)). Plotting the logarithm of the number of occupied boxes vs. the logarithm of the reciprocal of the unit size, the slope of the best fit line will correspond approximately to the Hausdorff-Besicovitch dimension of the object in a metric space (Figure 3), defined as

$$
D_{\mathrm{HB}}=\lim _{r \rightarrow 0} \frac{\log N(r)}{\log (1 / r)},
$$

where $N(r)$ is the number of boxes of size $r$ needed to cover the object. In some cases, this is akin to the Minkowski-Bouligand dimension, or Kolmogorov dimension, being this discussion however outside the scope of this communication. In simple words, the number of measuring units used, are proportional to their unit size, or similarity ratio, raised to the negative $D_{\mathrm{HB}}$ power.
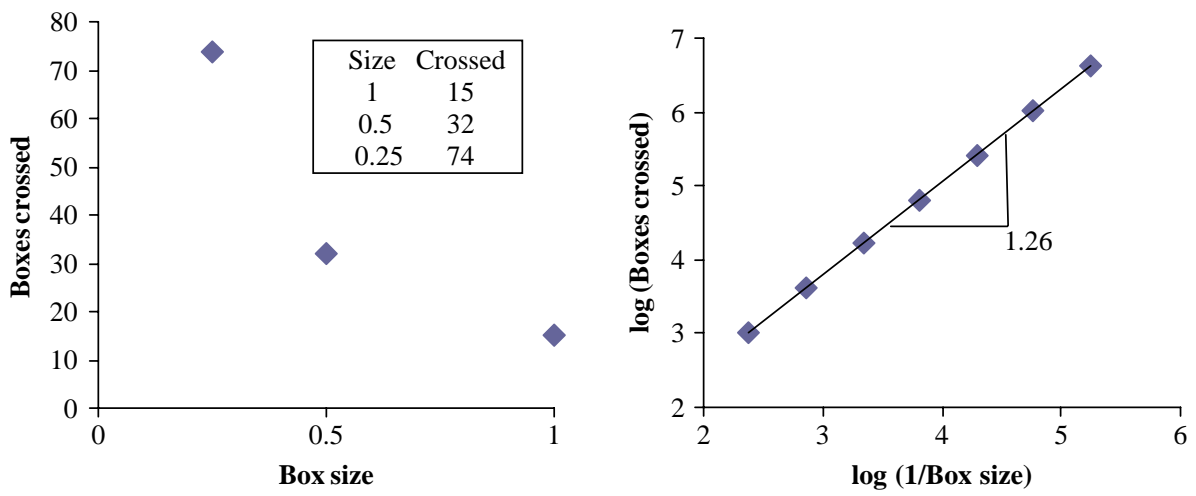

Figure 3. Box-counting method applied to the object in Figure 1(III). Entries on the left correspond to Figure 2(I)-(III). The complete analysis on the right may be carried out implementing the box-counting algorithm into a standard spreadsheet, or using one of the many freeware programs available on the internet. 
Thus, the path between points $\mathrm{A}$ and $\mathrm{B}$ in Figures 1(III) and 2(I)-(III) has a Hausdorff-Besicovitch dimension equal to the limit of the sequence (Figure 3),

$$
\frac{\log (1024)}{\log (243)}, \frac{\log (4096)}{\log (729)}, \frac{\log (16384)}{\log (2187)}, \ldots \Rightarrow \frac{\log \left(4^{n}\right)}{\log \left(3^{n}\right)}=\frac{n \log (4)}{n \log (3)}=\frac{\log (4)}{\log (3)}=1.26
$$

This procedure may be used for any entity in any metric space resulting in a quantity equivalent to a dimension. In fact, applied to the straight line in Figure 1(I), it takes one big box with size one, two with size $1 / 2$, four with size $1 / 4$ and so on, such that at the limit, $\log 2^{n} / \log 2^{n}=1$, while for the surface in Figure 1(II), one requires four boxes size 1/2, 16 boxes size $1 / 4$ and so on with $\log 2^{2 n} / \log 2^{n}=2$, as expected from Euclidean geometry. It must be noted though, that a very common mistake incurred in using this technique is the lack of attention for the limiting nature of Equation (1). Obviously, not all crooked objects are fractals. Often, ratios of logarithms are reported with too coarse box sizes and an erroneous conclusion for a fractal dimension is stated. Indeed, one of the drawbacks of the box-counting method is the fact that the series in Equation (1) may take a long time to converge for not so 'well-behaved' fractals.

Anyway, accordingly and for illustrative purposes, although both results for the straight line and the surface agree with the topological interpretation of a dimension, a common question is how to interpret a dimension equal to 1.26 which is neither 1 nor 2 . A simple interpretation may just conclude that the path in Figures 1(III) and 2(I)-(III) is something between a line and a plane, therefore with a dimension that is not a whole number but rather a fraction, or in short, it is a fractal. For a better definition, it may be said that a fractal is an object with a Hausdorff-Besicovitch dimension exceeding its smallest topological, or Euclidean, dimension.

Kinetic considerations readily stem from this geometric description [4,53] as much as the time to go from point A to B exceeds the one expected for the shortest path, either along a straight line or resulting from fixed tortuosity with a homogeneous diffusion medium. For a long time now, a heuristic connection has been established between the fractal dimensionality of Brownian motion in a heterogeneous space and the mean first-passage time through it [69]. The early models described discrete random walks on a lattice with transition probabilities drawn from a distribution with infinite variance, known as a Lévy distribution [27], although this mean first-passage time parameter may not be accurately defined, unlike for other higher moments. In fact, for a persistent process in a given microenvironment, the average amount of time spent there is infinite and a quantitative relation with its fractal dimension is impossible to establish. Instead, a quantitative relationship exists between the mean first-passage time to escape a given region of space and the Hausdorff-Besicovitch dimensionality.

In general, translationally invariant Markov processes are called Lévy processes, in one, two or three (Euclidean) dimensions, if their characteristic function is proportional to $\exp \left(-x^{h}\right)$, where $h$ is an 'heterogeneous' exponent for the trajectories of the process. Examples are, Brownian motion, Poisson processes, Feller-Markov processes, interlacing processes, among others. So, for a one-dimensional Lévy trajectory $x(t)$, the mean magnitude of displacement $\langle x(t)-x(0)\rangle$ is proportional to $t^{1 / h}$, for $t>0$. Therefore, the number of boxes of side $r$ required to cover the distance travelled between $t$ and $t+r$ is equal to

$$
\frac{\text { Distance covered }}{\text { Box size }}=\frac{r^{(1 / h)-1}}{r}=r^{(1 / h)-2} \text {. }
$$


Hence, the Hausdorff-Besicovitch dimension becomes $D_{\mathrm{HB}}=\log \left(r^{(1 / h)-2}\right) / \log (1 / r)=$ $2-1 / h$ [69], and for a Brownian motion $h=2$ and $D_{\mathrm{HB}}=3 / 2$ as expected.

As Kopelman put it long time ago, 'fractal differ from Euclidean spaces not only in their typical 'fractal' dimension $d_{f}$, but also in having more than one relevant dimension' [35]. Several definitions of dimension have been derived [63,71] what in some extent has created a bit of confusion among end users. As an example, particularly for the case of a random walker, such as a drug molecule, the recurrence probability $P$ of its returning to the origin after a time $t$ is defined as the spectral dimension $d_{\mathrm{s}}$ such that $P \sim t^{-d_{\mathrm{s}} / 2}$, also known as an anomalous waiting time distribution. Fractal spaces have characteristically $d_{\mathrm{s}}<d_{\mathrm{f}}<d$, where $d$ stands for the dimension of the Euclidean spaces in which they are embedded [49], while for these $d_{\mathrm{s}}=d_{\mathrm{f}}=d$, meaning that all locations are available for 'walking'. In kinetic terms, Smoluchowski was perhaps the first, back in 1917, to point out that, for low dimensions, diffusion-limited reactions have rate constants and corresponding diffusion coefficients that depend on the time scale [70]. In fact, he replaced the classical term 'mean free path' under such conditions, by 'diffusion length' of the random walker, defining what became to be known as Smoluchowski kinetics.

\section{Diffusion in fractal spaces}

Considering a drug molecule moving randomly and under no constraints, its probability density as a function of distance and time satisfies the condition known as Fick's second law of diffusion. However, under anomalous diffusion, instead of the mean square displacement being proportional to time, as observed in Gaussian processes, such as Fickean diffusion, it increases slower or faster than linearly with time, becoming $\left\langle x(t)^{2}\right\rangle \propto t^{2 / h}[10,41]$, according to an anomalous diffusion exponent $h>1$, but different from 2 , the Fickean value $\left(h=2 / d_{\mathrm{s}}\right)$.

For an irreversible process, by a thermodynamic linear approximation, the macroscopic flux of an extensive quantity, such as mass, across a fractal interface is described by the generalized diffusion equation, also referred to as the fractional diffusion Equation $[19,41]$,

$$
\frac{\partial^{(2 / h)}}{\partial t^{(2 / h)}} M(t)=k \cdot \Delta C(t),
$$

where $k$ is a rate constant, $\Delta C(t)$ is a time dependent driving force, such as a concentration gradient and $\partial^{\nu} / \partial t^{\nu}$ stands for the Riemann-Liouville fractional derivative operator, being $\nu$ any real number (actually, a differintegral defined as $\partial^{\nu} y / \partial t^{\nu}=(1 / n !)(\mathrm{d} / \mathrm{d} t)$ $\int_{0}^{t}\left(y(\zeta) /(t-\zeta)^{n}\right) \mathrm{d} \zeta$ ). In fact, fractional differential Equations (or fractional-order differential equations), such as Equation (3), allow the exploration of various boundary conditions relevant for many physical and biological phenomena. Particularly, biological systems deviate from the asymptotic power law for very small scales, and fractional calculus allows the interpolation between the macro and the microenvironment. It can be dated back to Leibniz's correspondence about non-integer differentiation, being today applied to numerous phenomenological theories in physics, chemistry and biology [64]. It generalizes the derivative and antiderivative operations from integer orders to the entire complex plane.

Accordingly, for the simplest case of a one-dimensional diffusion from a fractal interface,

$$
\frac{\partial^{(3-h)} C(x, t)}{\partial t^{(3-h)}}=D_{h} \frac{\partial^{2} C(x, t)}{\partial x^{2}}, \quad 2 \leq h<3,
$$


where for $h=2$ the classical Fick's second law of diffusion results. $D_{h}$ corresponds to the fractional diffusivity, which may be time dependent due to the fractal nature of the diffusional interface,

$$
D_{h}(t)=D_{h} \frac{t^{(2-f)}}{\Gamma(3-f)},
$$

(some authors prefer the notation $n !=\Gamma(1-n)$, where $\Gamma$ refers to the Gamma function). Furthermore, the flux across the fractal interface becomes,

$$
J_{f}(x, t)=\frac{\partial}{\partial t}\left(D_{h}(t) * \frac{\partial C(x, t)}{\partial x}\right)
$$

where $*$ refers to the convolution operator [19].

In more general terms, the so called fractional kinetic equation, first introduced by Zaslavsky [64,85], reads

$$
\frac{\partial^{h}}{\partial t^{h}} P(x, t)=\frac{\partial^{n}}{\partial x^{n}} P(x, t)+g(x, t)
$$

where the probability density $P(x, t) \geq 0$, for finding a given particle at position $x$ at any time $t$, satisfies the usual normalization condition,

$$
\int_{-\infty}^{\infty} P(x, t) \mathrm{d} x=1 \quad(t>0)
$$

but also the identity condition,

$$
\int_{-\infty}^{\infty} \frac{\partial^{n}}{\partial x^{n}} P(x, t) \mathrm{d} x=0
$$

having been shown that to ensure positivity $(P(x, t) \geq 0), 0<n \leq 2$ [54]. Again, for the usual case of Fickean diffusion, $n=2$ and with the source function $g(x, t)$ in Equation (7) assumed to be $\delta(x) \cdot \delta(t)$ (where $\delta(\cdot)$ corresponds to the Dirac delta function), then

$$
\frac{\partial}{\partial t} P(x, t)=\frac{\partial^{2}}{\partial x^{2}} P(x, t)
$$

with initial condition $P(x, t=0)=\delta(x)$, as in Fick's second law of diffusion.

However, in fractal environments, space restricted, the fractional derivative in Equation (7) corresponds to a non-local operator for the probability density of a symmetrically wandering particle from $x=0$ at $t=0$,

$$
\frac{\partial^{h}}{\partial t^{h}} P(x, t)=\frac{\partial^{n}}{\partial x^{n}} P(x, t)+g(t) \delta(x),
$$

defining now the source function $g(x, t)=g(t) \cdot \delta(x)$. Integrating Equation (11) with respect to $x$ between $-\infty$ and $+\infty, g(t)$ is found to be

$$
g(t)=\frac{t^{-h}}{\Gamma(1-h)}
$$


using the fractional differentiation rule

$$
\frac{\partial^{\nu}}{\partial t^{\nu}} y(x)=y(x) * \frac{t_{+}^{-(\nu+1)}}{\Gamma(-\nu)},
$$

in the regularized form for $0<\nu<1$, to avoid the singularity near to the upper limit of integration, where $*$ stands for the convolution operation, $\Gamma(\cdot)$ is the Gamma function and the subscript + corresponds to the truncation function

$$
y_{+}(x)= \begin{cases}y(x), & x>0 \\ 0, & x<0\end{cases}
$$

Thus, the fractional kinetic equation becomes,

$$
\frac{\partial^{m}}{\partial t^{m}} P(x, t)=\frac{\partial^{n}}{\partial x^{n}} P(x, t)+\frac{t^{-h}}{\Gamma(1-h)} \delta(x) .
$$

The case of $0<h<1$ and $n=2$ corresponds to a fractal Brownian motion with a mean square displacement equal to

$$
\left\langle x^{2}\right\rangle=\frac{2}{\Gamma(h+1)} t^{h}
$$

\section{Fractal kinetics}

For the simple diffusion-limited reaction $\mathrm{A}+\mathrm{A} \rightarrow \mathrm{A}_{2}$, Kopelman showed that its rate is proportional to $t^{-h} \times[\mathrm{A}]^{2}$, where $0<h<1$ depends on the dimensionality of the medium [34]. If the reaction can only occur on a one-dimensional channel, then $h=0.5$; in full three dimensions $h=0$ according to the law of mass action; and in a percolation cluster, as an example of a heterogeneous medium with topological constraints, $h=1 / 3$. This way, time-dependent rate constants have become the hallmark of fractal kinetics, although not always fully documented.

In an earlier work, Savageau proposed a power law formalism, also known as the generalized mass action kinetics, for the rate of a multireactant process in the intracellular space [65] such that,

$$
\text { Rate }=k \prod_{i=1}^{n}\left[\mathrm{~A}_{i}\right]^{a_{i}}
$$

where $k$ is explicitly time independent and the kinetic orders $a_{i}$ correspond to the molecularity of the single step reaction between $n$ reactants, under the classical law of mass action $[66,67]$. Basically, reactant concentrations are raised to non-integer powers instead of deriving time dependent rate constants. It has been extensively assumed, particularly in the biochemical literature, that this phenomenological approximation is equivalent to Kopelman's time-dependence $[7,15,18]$. However, although it may be applied in many cases, it fails to describe others more complex, such as saturation and sigmoidicity [24]. Schnell et al. showed recently that this correspondence is only valid for long time and under the special case of a reaction $\mathrm{A}+\mathrm{B} \rightarrow \mathrm{AB}$ with $\mathrm{A}_{0}=\mathrm{B}_{0}$, equivalent to a homodimeric reaction, not that general in nature $[23,68]$. The fractal 
kinetics based ordinary differential equations (ODE) are not universally equivalent to their power law approximation. Unfortunately, both Kopelman and Savageau coin their work as 'fractal kinetics' mostly because they evolve from an underlying fractal geometry conception, in spite of the significantly different mathematical outcomes. Nevertheless, fractal kinetics and time-dependent rate constants seem to be the most adequate mechanistic and observational theory when diffusion-limited processes are relevant. That's why fractional calculus should be taken into consideration. A recent paper by Brauers et al. [11] clarifies things considerably. Summarizing their rationale, for any given decaying phenomenon, let's say a finite amount of drug in an open system, the most elemental kinetic relationship between its rate $(\mathrm{d} M / \mathrm{d} t)$ and its driving force $(M(t))$ is

$$
\frac{\mathrm{d} M}{\mathrm{~d} t}=-k M(t)^{n}
$$

where $k$ corresponds to a (proportionality) rate constant and $n$ is the overall order of the process. For zero-order processes $n=0$ and the rate is fixed, while for subsequent integer orders the molecular reaction rationale is followed. The integrated solution for Equation (16) becomes

$$
M(t)=M_{\mathrm{o}}\left(1+(n-1) M_{0}^{n-1} k t\right)^{\frac{1}{1-n}}
$$

known as the generalized Pareto function with initial condition $M(t=0)=M_{\mathrm{o}}$. Making use of the deformed $q$-exponential function introduced by Tsallis [74], denoted as $\mathrm{e}_{q}^{x}=$ $(1+(1-q) x)^{1 /(1-q)}$ for $1+(1-q) x>0$ and 0 otherwise (note: $\mathrm{e}_{1}^{x}=\mathrm{e}^{x}$ ), Equation (17) may assume the algebraically equivalent form,

$$
M(t)=M_{\mathrm{o}} \mathrm{e}_{n}^{-t / \tau_{n}}
$$

also referred to as a stretched exponential, with the order-dependent time constant, or characteristic time, $\tau_{n}=\left(M_{0}^{n-1} k\right)^{-1}$. By differentiating Equation (18), it may be parameterized in terms of a time-dependent rate coefficient,

$$
\begin{aligned}
\frac{\mathrm{d} M}{\mathrm{~d} t}=-\frac{M_{\mathrm{o}}}{\tau_{n}}\left(\mathrm{e}_{n}^{-\frac{t}{\tau_{n}}}\right)^{n} & =-\frac{M_{\mathrm{o}}}{\tau_{n}}\left(1+(n-1) \frac{t}{\tau_{n}}\right)^{\frac{1}{1-n}-1} \\
& =-\boldsymbol{\kappa}(t) M(t),
\end{aligned}
$$

where $\boldsymbol{\kappa}(t)=\left(\tau_{n}+(n-1) t\right)^{-1}$. The net result is that for $t \ll \tau_{n}$ the effective rate gets slowed down, while for $t \gg \tau_{n}$ and $n=1$ the classical first-order exponential behaviour results.

Recognizably, for those early times and more complex systems, this does not apparently exhibit a power law relationship useful for describing experimental data. However, for non-extensive (non-additive) variables, i.e. not proportional to size, occurring in a dimension $d$, relaxation theory shows that this is a particular case of the general expression derived as [12],

$$
M(t)=M_{\mathrm{o}} \mathrm{e}_{n}^{-k(t) M_{\mathrm{o}}^{n-1} t^{d}}=M_{\mathrm{o}}\left(1+(n-1) k(t) M_{0}^{n-1} t^{d}\right)^{\frac{1}{1-n}},
$$


which is the solution to the fractional differential equation,

$$
\frac{\mathrm{d}^{d} M}{\mathrm{~d} t^{d}}=-\boldsymbol{\kappa}_{d}(t) \cdot M(t)^{n}
$$

Now, the effective rate constant

$$
\mathbf{\kappa}(t)=\frac{t^{d-1} d}{\tau_{n}^{d}}\left(1+(n-1)\left(\frac{t}{\tau_{n}}\right)^{d}\right)^{-1}
$$

where

$$
\tau_{n}=\left(M_{0}^{n-1} k(t)\right)^{-1 / d}
$$

clearly reveals two asymptotic characteristics: as $t \rightarrow 0 \mathbf{\kappa}(t) \propto t^{d-1}$, while as $t \rightarrow \infty$ one recognizes the homogeneous case depicted above $\mathbf{\kappa}(t)=t^{-1}$. Identifying Kopelman's fractal parameter $0<h<1$ as $1-d$, one may recognize the phenomenological characteristics of fractal kinetics associated with non-homogeneous microenvironments.

Characteristically, for $n=1$ and $d=1$,

$$
\frac{\mathrm{d} M}{\mathrm{~d} t}=-k M(t) \quad \text { and } M(t)=M_{\mathrm{o}} \mathrm{e}^{-k t},
$$

corresponding to the classical first-order homogeneous kinetics. For $n=1$ and $d \neq 1$,

$$
\frac{\mathrm{d}^{d} M}{\mathrm{~d} t^{d}}=-k M(t) \quad \text { and } \quad M(t)=M_{\mathrm{o}} \mathrm{e}^{-k t^{d}},
$$

corresponding to a Weibull heterogeneous kinetics, or a stretched exponential kinetics if $0<d<1$, and for $n=2$ and $d \neq 1$,

$$
\frac{\mathrm{d}^{h} M}{\mathrm{~d} t^{h}}=-k M(t)^{2} \quad \text { and } \quad M(t)=M_{\mathrm{o}}\left(1+M_{\mathrm{o}}(k t)^{h}\right)^{-1}
$$

one obtains the generalized second-order kinetics relationships.

\section{Compartmental modelling}

Observed concentration versus time profiles with long-time decaying tails, $C(t) \propto t^{-\lambda}$ for $t>\tau$, where $\tau$ is to the threshold time for the decay, were first described by negative power laws, sequential power laws and gamma functions $\left(C(t)=k t^{-\lambda} \mathrm{e}^{-\alpha t}\right)[6,26,57,81,82]$.

As discussed above, in fractal microenvironments kinetic rate constants become timedependent $\left(k(t)=k t^{-h}\right)$ with a fractal exponent $h=1-\left(d_{\mathrm{s}} / 2\right)$. At the molecular level, the spectral dimension $d_{\mathrm{s}}$ characterizes the random walk of a molecule in the fractal space [1], assuming the classical value of 2 in fully unrestricted homogeneous spaces [4]. Thus, the notion of time-dependent rate constants has been used in the context of compartmental and non-compartmental PK, although it bears also interesting potential in the context of system analysis and input-output convolution relationships, as hinted above.

In fact, using the simplest open compartmental model with instantaneous input (e.g. bolus dosing), the implementation of a fractal disposition in terms of the mass of drug, 
is reasonably straight forward. For a zero-order time-dependent elimination,

$$
\frac{\mathrm{d} M}{\mathrm{~d} t}=-K_{0} t^{-h^{\text {integrating }}} \stackrel{M}{\rightarrow} \int_{M_{\mathrm{o}}}^{M} \mathrm{~d} M=-K_{0} \int_{0}^{t} t^{-h} \mathrm{~d} t \rightarrow M=M_{\mathrm{o}}\left(\frac{K_{0}}{h-1}\right) t^{(1-h)}
$$

and for a first-order time-dependent elimination,

$$
\frac{\mathrm{d} M}{\mathrm{~d} t}=-k t^{-h} M \stackrel{\text { integrating }}{\rightarrow}|\ln M|_{M_{\mathrm{o}}}^{M}=\frac{-k}{1-h}\left|t^{1-h}\right|_{0}^{t} \rightarrow M=M_{\mathrm{o}} \mathrm{e}^{-\frac{k}{1-h} t^{(1-h)}}
$$

However, particularly when a drug reveals in vivo fractal PK characteristics, such as nonloglinear terminal elimination, the single compartment model falls short from describing the anticipated distribution delays into the whole body. For the same reasons argued in 'homogeneous' PK, drugs may require some time to distribute into less irrigated tissues or those with less favourable partition coefficients, eliciting excessive initial blood concentrations in comparison to a single exponential back extrapolation towards time zero. The instantaneous distribution assumption becomes thus unusable, being the next degree of complexity, under the compartmental approach, the conception of a bicompartmental disposition. Furthermore, drugs with localized sites of action, or biophases, for which local concentrations are better indicators of drugs efficacy, and/or toxicity, in comparison to blood levels, benefit considerably from a multicompartmental modelling approach.

Unfortunately, this simple move from a one to a two compartment model in a fractal PK context generates significant complexity. Fuite et al. were the first to tackle the problem [22], complemented later in different ways [14,51]. Since the original work focused on the drug mibefradil and its fractal hepatic metabolism, a two compartment open model with peripheral elimination only was chosen $\left(k_{10}=0\right.$ in Figure 4$)$. The more common central elimination assumption $\left(k_{20}=0\right.$ in Figure 4$)$ was investigated later [51]. Either way, following the standard law of mass action, a system of linear ODE's results with constant coefficients for classical homogeneous PK, since all rate constants are fixed, analytically solvable by Laplace transformation and the partial fractions theorem, or Heaviside's expansion.

Allowing some rate constants to be time-dependent, the model becomes a system of linear ODE's with variable coefficients, for which no analytical solutions are usually found. The usual two compartments mamillary model (Figure 4 with $k_{20}=0$, for central elimination only), assuming a fractal distribution rate constant between the central and the peripheral compartment $\left(k_{12}(t)=k_{12} t^{-h}\right.$; for the sake of simplicity, redistribution and elimination will be assumed to be time independent at this point), becomes

One simple trick to handle such a system of first-order ODE's is to differentiate the first equation again, replace the $M_{2}$ derivative with the second equation and extract $M_{2}$ from the first one to replace it as well. This way a second-order heterogeneous ODE in $M_{1}$

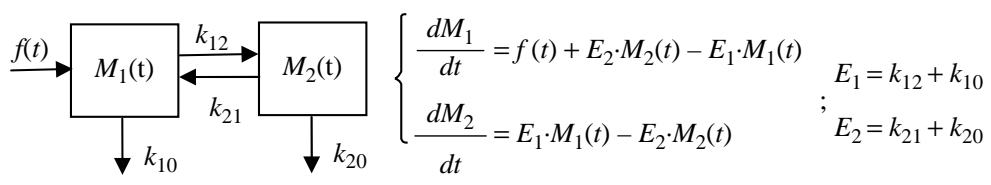

Figure 4. Fully connected two compartments open model with first-order mass transfer and input $f(t)$, where $E_{i}$ correspond to the overall exit rate constant from compartment $i$. The catenary case with $k_{10}=0$ was used by Cheminiak et al. [14] and Fuite et al. [22] with $k_{20}(t)=k_{20} \cdot t^{-h}$, while Marsh et al. [51] tested also the $k_{20}=0$ mamillary case with $k_{21}(t)=k_{21} \cdot t^{-h}$. 
alone is obtained as

$$
\frac{\mathrm{d}^{2} M_{1}}{\mathrm{~d} t^{2}}+\left(k_{21}+k_{10}+k_{12} t^{-h}\right) \frac{\mathrm{d} M_{1}}{\mathrm{~d} t}+\left(k_{21} k_{10}-k_{12} h t^{-(h+1)}\right) M_{1}(t)=k_{21} f(t)+\frac{\mathrm{d} f}{\mathrm{~d} t},
$$

with the corresponding homogeneous case (not to be confused with the notion of homogeneity used above for the compartment concept and biological microenvironments at large),

$$
\frac{\mathrm{d}^{2} M_{1}}{\mathrm{~d} t^{2}}+\left(k_{21}+k_{10}+k_{12} t^{-h}\right) \frac{\mathrm{d} M_{1}}{\mathrm{~d} t}+\left(k_{21} k_{10}-k_{12} h t^{-(h+1)}\right) M_{1}(t)=0,
$$

when $f(t)=0$, such as with a bolus administration.

Either formulation of this variable coefficients problem is hard to solve analytically, or just insolvable depending on the nature of those coefficients. The latter was worked on by Fuite et al. [22], who resorted to a perturbation numerical method to solve their model, while Marsh et al. [51] proposed a simulated annealing technique for both the catenary and the mamillary models. Very unfortunately, Chelminiak et al. [14] transcribed wrongly Fuite's model incurring in an erroneous analysis just recently identified [60]. Nevertheless, their attempt suggests valuable analytical approximations again to mitigate the aforementioned difficulty.

One more useful exercise consists in adding both equations in Figure 5, which results in

$$
\frac{\mathrm{d} M_{1}}{\mathrm{~d} t}+\frac{\mathrm{d} M_{2}}{\mathrm{~d} t}=f(t)-k_{10} M_{1}(t)
$$

and integrating with respect to $t$,

$$
M_{1}(t)+M_{2}(t)=\int_{0}^{t} f(u) \mathrm{d} u-k_{10} \int_{0}^{t} M_{1}(u) \mathrm{d} u+c_{0} .
$$

Then, solving the second differential equation for $M_{2}$ one obtains,

$$
M_{2}(t)=\mathrm{e}^{-k_{21} t}\left[c_{1}+k_{12} \int_{0}^{t} u^{-h} \mathrm{e}^{k_{21} u} M_{1}(u) \mathrm{d} u\right]
$$

which, replaced into Equation (32), results in the following Volterra integral expression,

$$
M_{1}(t)+k_{10} \int_{0}^{t} M_{1}(u) \mathrm{d} u+k_{12} \int_{0}^{t} u^{-h} \mathrm{e}^{-k_{21}(t-u)} M_{1}(u) \mathrm{d} u=\int_{0}^{t} f(u) \mathrm{d} u+c_{0}+c_{1} \mathrm{e}^{-k_{21} t},
$$

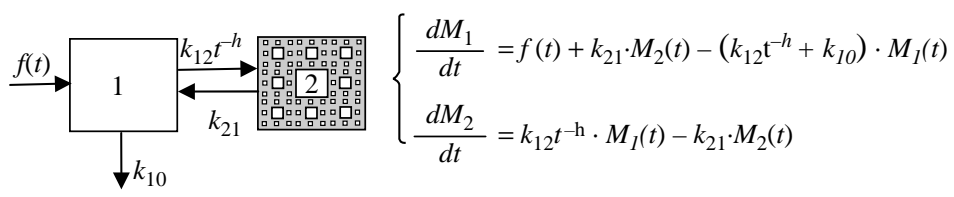

Figure 5. Two compartments mamillary model with just one fractal kinetic step, namely from the central to the peripheral compartments. 
being constants $c_{1}=-M_{2}(0)$ and $c_{0}=M_{1}(0)+M_{2}(0)$. Differentiating it twice, Equation (29) readily results. This implicit solution is amenable to describe experimental concentration data $\left(C_{1}\right)$ resulting, for instance, from a bolus dose $D$, in the form,

$$
C_{1}(t)+k_{10} A U C_{0}^{t}+\left(k_{12} \mathrm{e}^{-k_{21} t}\right) *\left(t^{-h} C_{1}(t)\right)=\frac{D}{V_{1}},
$$

using $V_{1}$ for the apparent volume of distribution and the $*$ convolution notation for

$$
x(t)^{*} y(t)=\int_{0}^{t} x(t-u) y(t) \mathrm{d} u
$$

or, numerically with a small step size $\Delta t$,

$$
\cong \Delta t \sum_{i=0}^{n-1}\{x[t-(i+0.5) \Delta t] y[(i+0.5) \Delta t]\},
$$

with $n=t / \Delta t$ (note: for actual calculations better numerical convolution algorithms are found implemented in software such as Matlab, S-Plus and R).

A similar derivation may be carried out focusing on the $M_{2}(t)$ variable, the amount of drug in the peripheral fractal compartment according to Figure 5, in which case,

$$
\frac{\mathrm{d}^{2} M_{2}}{\mathrm{~d} t^{2}}+\left(k_{21}+k_{10}+k_{12} t^{-h}+h t^{-1}\right) \frac{\mathrm{d} M_{2}}{\mathrm{~d} t}+\left(k_{10}+h t^{-1}\right) k_{21} M_{2}(t)=k_{12} t^{-h} f(t) .
$$

However, one major problem with 'fractal-like' variable coefficients is the definition of the ODE's at the singularity $t=0$, since then, $t^{-h}$ becomes indefinite. A consensual recommendation $[51,68]$ seems to be the redefinition of the time-dependent rate constants in terms of the Zipf-Mandelbrot distribution,

$$
k(t)=\frac{k}{(t+\tau)^{h}}, \quad 0 \leq h \leq 1,
$$
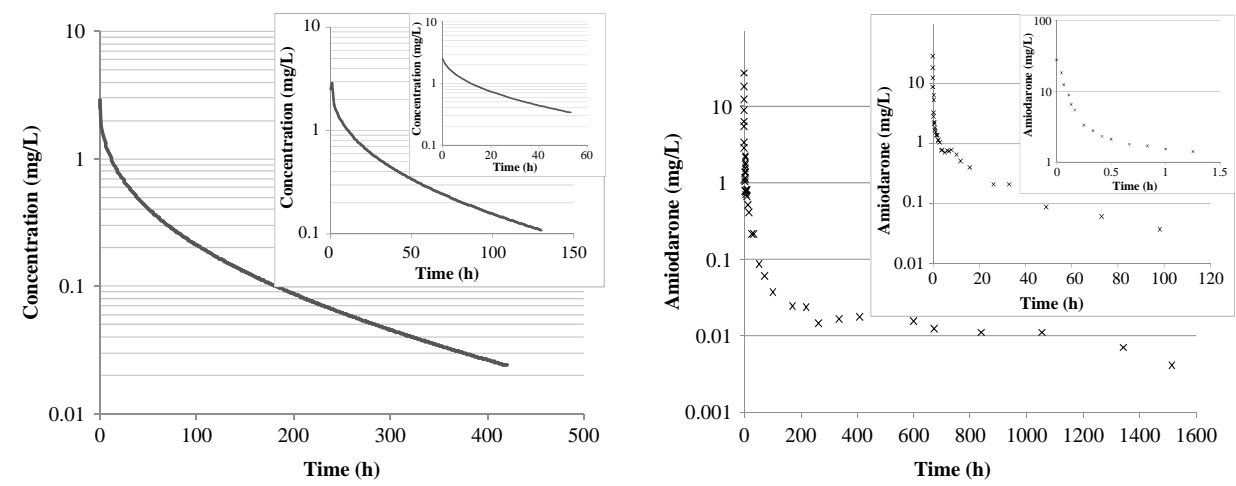

Figure 6. Left panel, simulated profiles according to Equation (35) with $k_{10}=0.001 \mathrm{~h}^{-1}$, $k_{12}=0.2 \mathrm{~h}^{-1}, k_{21}=0.02 \mathrm{~h}^{-1}, h=0.6, D=500 \mathrm{mg}$ and $V_{1}=200 \mathrm{~L}$. Right panel, amiodarone postinfusion data kindly supplied by Prof. G.T. Tucker, referring to a $10 \mathrm{~min}$ i.v. infusion of a $400 \mathrm{mg}$ dose administered to human subjects and followed up for over 60 days [76]. 
where $\tau$ is a small positive constant defined as the critical time for the onset of the fractal process. This way, Equation (30) becomes

$$
\frac{\mathrm{d}^{2} M_{1}}{\mathrm{~d} t^{2}}+\left(k_{21}+k_{10}+k_{12}(t+\tau)^{-h}\right) \frac{\mathrm{d} M_{1}}{\mathrm{~d} t}+\left(k_{21} k_{10}-k_{12} h(t-1) t^{-(h+1)}\right) M_{1}(t)=0
$$

with better numerical characteristics.

To solve this kind of expressions, an analytical method recently proposed by Li [43] may be used, involving a Volterra integral for the initial value problem, or a Fredholm integral for the boundary value problem. It may even be extended to higher-order linear ODE's. Essentially, he showed that, based on the existence of a unique solution and integrating twice Equation (38) in the form $M_{1}^{\prime \prime}(t)+p(t) M_{1}^{\prime}(t)+q(t) M_{1}(t)=g(t)$, an implicit solution in $M_{1}$ may be found as,

$$
M_{1}(t)+\int_{0}^{t}\left[p(u)+(t-u)\left(q(u)-p^{\prime}(u)\right) M_{1}(u)\right] \mathrm{d} u=\phi(t),
$$

where

$$
\phi(t)=M_{1}(0)+\left[M_{1}(0) p(0)+M_{1}^{\prime}(0)\right] t+\int_{0}^{t}(t-u) g(u) \mathrm{d} u
$$

with initial conditions $M_{1}(0)$ and $M_{1}^{\prime}(0)$. This standard Volterra integral with a continuous kernel may be solved using first a Taylor series expansion with a second-order approximation (according to the desired degree of approximation), meaning truncated after the third term,

$$
M_{1}(t) \approx M_{1}(\tau)-M_{1}^{\prime}(\tau)(\tau-t)+\frac{1}{2} M_{1}^{\prime \prime}(\tau)(\tau-t)^{2}
$$

which replaced into the integrand in Equation (51) results in,

$$
V_{02}(t) M_{1}^{\prime \prime}(t)+V_{01}(t) M_{1}^{\prime}(t)+V_{00}(t) M_{1}(t)=\phi(t)
$$

where

$$
\begin{gathered}
V_{02}(t)=\frac{1}{2}\left[p(0) t^{3}+\int_{0}^{t}(t-\tau)^{3} q(\tau) \mathrm{d} \tau\right]-\int_{0}^{t}(t-\tau)^{2} p(\tau) \mathrm{d} \tau \\
V_{01}(t)=-p(0) t^{2}-\int_{0}^{t}(t-\tau)^{2} q(\tau) \mathrm{d} \tau+\int_{0}^{t}(t-\tau) p(\tau) \mathrm{d} \tau \\
V_{00}(t)=1+p(0) t-\int_{0}^{t}(t-\tau) q(\tau) \mathrm{d} \tau .
\end{gathered}
$$

To finally estimate $M_{1}(t)$, Li derived a third equation in $M_{1}^{\prime \prime}, M_{1}^{\prime}$ and $M_{1}$, by two different approaches, in order to set with Equations (38) and (41), a solvable three equations system with three unknowns. By his first approach, denoted as the differentiation method,

$$
V_{12}(t) M_{1}^{\prime \prime}(t)+V_{11}(t) M_{1}^{\prime}(t)+V_{10}(t) M_{1}(t)=\phi(t)
$$


where

$$
\begin{gathered}
V_{12}(t)=\frac{1}{2}\left[p(0) t^{2}+\int_{0}^{t}(t-\tau)^{2} q(\tau) \mathrm{d} \tau\right]-\int_{0}^{t}(t-\tau) p(\tau) \mathrm{d} \tau \\
V_{11}(t)=1-p(0) t-\int_{0}^{t}(t-\tau) q(\tau) \mathrm{d} \tau+\int_{0}^{t} p(\tau) \mathrm{d} \tau \\
V_{10}(t)=p(0)+\int_{0}^{t} q(\tau) \mathrm{d} \tau
\end{gathered}
$$

Finally,

$$
M_{1}(t)=\frac{\operatorname{det}\left[\begin{array}{ccc}
\phi(t) & V_{01}(t) & V_{02}(t) \\
\phi^{\prime}(t) & V_{11}(t) & V_{12}(t) \\
g(t) & p(t) & 1
\end{array}\right]}{\operatorname{det}\left[\begin{array}{ccc}
V_{00}(t) & V_{01}(t) & V_{02}(t) \\
V_{10}(t) & V_{11}(t) & V_{12}(t) \\
q(t) & p(t) & 1
\end{array}\right]},
$$

where

$\operatorname{det}\left|\begin{array}{lll}x_{11} & x_{12} & x_{13} \\ x_{21} & x_{22} & x_{23} \\ x_{31} & x_{32} & x_{33}\end{array}\right|=x_{11}\left|\begin{array}{ll}x_{22} & x_{23} \\ x_{32} & x_{33}\end{array}\right|-x_{12}\left|\begin{array}{ll}x_{21} & x_{23} \\ x_{31} & x_{33}\end{array}\right|+x_{13}\left|\begin{array}{ll}x_{21} & x_{22} \\ x_{31} & x_{32}\end{array}\right|$ and $\operatorname{det}\left|\begin{array}{ll}a & b \\ c & d\end{array}\right|=a d-c b$

For the model in Figure 5 and according to Equation (37), $p(t)=k_{21}+k_{10}+$ $k_{12}(t+\tau)^{-h}, q(t)=k_{21} k_{10}-k_{12} h(t-1) t^{-(h+1)}$ and $f(t)=D \delta(t)$, with $\delta(t)$ standing for the Dirac delta function, initial conditions $M_{1}(0)=D$, the bolus i.v. dose, and $M_{1}^{\prime}(0)=-D\left(k_{10}+k_{12} \tau^{-h}\right)$. Then, using the $*$ convolution notation,

$$
\begin{gathered}
V_{02}(t)=\frac{1}{2}\left[\left(k_{21}+k_{10}\right) t^{3}+t^{3} * q(t)\right]-t^{2} * p(t), \\
V_{01}(t)=-\left(k_{21}+k_{10}\right) t^{2}-t^{2} * q(t)+t * p(t), \\
V_{00}(t)=1+\left(k_{21}+k_{10}\right) t-t * q(t), \\
V_{12}(t)=\frac{1}{2}\left[\left(k_{21}+k_{10}\right) t^{2}+t^{2} * q(t)\right]-t * p(t), \\
V_{11}(t)=1-\left(k_{21}+k_{10}\right) t-t * q(t)+\int_{0}^{t} p(u) \mathrm{d} u, \\
V_{10}(t)=\left(k_{21}+k_{10}\right)+\int_{0}^{t} q(u) \mathrm{d} u .
\end{gathered}
$$

For the constant terms in $p(t)$ and $q(t)$, using the distributive property of all linear operators, the following convolution rule for any constant $k$ and integer $n$ may be simply 
implemented,

$$
t^{n} * k=\int_{0}^{t} u^{n} k \mathrm{~d} u=\frac{k}{n+1} t^{n+1}
$$

while for the terms involving two time functions of the kind $t^{n} * t^{-h}$ a Laplace transformation is required which results in,

$$
t^{n} * t^{-h} \Rightarrow \mathrm{L}\left\{t^{n}\right\} \mathrm{L}\left\{t^{-h}\right\}=\frac{n ! \Gamma(1-h)}{s^{(n+2-h)}} \stackrel{\mathrm{L}^{-1}}{\Rightarrow} \frac{n ! \Gamma(1-h)}{\Gamma(n+2-h)} t^{n+1-h}
$$

where $\Gamma(\cdot)$ stands for the Gamma function and $\Gamma(n)=(n-1)$ ! for any positive integer $n$.

Alternative to the method of deriving a second-order ODE in just one variable by substitution, or when that renders a numerically unfavourable result, the linear system of differential equations with variable coefficients described in Figure 5 may still be solvable directly. One of the earliest proposals was made some time ago by Yamamoto [84] for a homogeneous system of the form

$$
\mathbf{M}^{\prime}=\mathbf{A}(t) \cdot \mathbf{M},
$$

where for the fully connected two ODE's system in Figure 4, with instantaneous input,

$$
\mathbf{M}^{\prime \mathrm{T}}=\left(\frac{\mathrm{d} M_{1}(t)}{\mathrm{d} t}, \frac{\mathrm{d} M_{2}(t)}{\mathrm{d} t}\right), \quad \mathbf{M}^{\mathrm{T}}=\left(M_{1}(t), M_{2}(t)\right),
$$

and the continuously differentiable coefficient matrix

$$
\mathbf{A}=\left(\begin{array}{cc}
-E_{1}(t) & E_{2}(t) \\
E_{1}(t) & -E_{2}(t)
\end{array}\right)
$$

He showed that this system may be reduced to another one with a constant coefficients matrix $\mathbf{B}$ and thus easier to solve,

$$
\mathbf{Y}^{\prime}=\mathbf{B} \cdot \mathbf{Y}
$$

under the transformation $\mathbf{M}=\mathrm{e}^{\mathbf{S} t} \mathbf{Y}$, if and only if the constant matrix $\mathbf{S}$ satisfies the conditions,

$$
\mathbf{A}^{\prime}(t)=\mathbf{S A}(t)-\mathbf{A}(t) \mathbf{S} \text { and } \mathbf{A}(0)=\mathbf{S}+\mathbf{B} .
$$

The challenge then becomes the estimation of matrices $\mathbf{S}$ and $\mathbf{B}$, particularly, if the fundamental matrix of the system is hard to find. This is defined as,

$$
\boldsymbol{\Phi}(t)=\mathrm{e}^{\mathrm{S} t} \cdot \mathrm{e}^{\mathbf{B} t},
$$

with its determinant known as the Wronskian and it corresponds to the non-singular matrix whose columns are the linearly independent solutions to the homogeneous Equation (52). The same approach may be extended to heterogeneous ODE's systems, such as $\mathbf{M}^{\prime}=\mathbf{A}(t) \cdot \mathbf{M}+\mathbf{r}(t)$, for which the solution to the initial valued problem is presented as,

$$
\mathbf{M}(t)=\boldsymbol{\Phi}(t) \boldsymbol{\Phi}(0)^{-1} \mathbf{M}(0)+\boldsymbol{\Phi}(t) \int_{0}^{t} \boldsymbol{\Phi}(u)^{-1} \mathbf{r}(u) \mathrm{d} u,
$$


with the requirement that the fundamental matrix $\boldsymbol{\Phi}(t)$ is integratable and inversable [17]. In general, there are no explicit methods to construct a fundamental matrix. However, if one solution is known, the dimension of the initial ODE's system may be reduced by one using the method of d'Alembert reduction. As an example, the previously discussed methodology could be used to estimate $M_{1}(t)$ being then the perhaps more elusive $M_{2}(t)$ estimated this way with added statistical advantages, since then the eigenvalues and eigenvectors of the original coefficient matrix would become known.

Still, a different method was proposed at about the same time by Vasilach [78] making use of composition algebras. For the heterogeneous system, in his notation to facilitate the interpretation,

$$
\frac{\mathrm{d} M_{i}}{\mathrm{~d} t}=r_{i}(t)+\sum_{j=1}^{n} a_{i j}(t) M_{i}(t), \quad 1 \leq i, j \leq n,
$$

the solution may be derived to be,

$$
M_{i}(t)=\sum_{j=1}^{n}\left(\int_{0}^{t} \mathrm{Z}_{i j}(t, u) r_{j}(u) \mathrm{d} u\right)+\sum_{j=1}^{n} Z_{i j}\left(t, t_{0}\right) M_{j}\left(t_{0}\right), \quad 1 \leq i, j \leq n
$$

or, for the homogeneous case, the same just omitting the term $r_{j}(u)$, being

$$
Z_{i j}\left(t, t_{0}\right)=\left\{\begin{array}{ll}
1+\int_{t_{0}}^{t} G_{i j}(t, u) \mathrm{d} u, & \text { for } i=j \\
\int_{t_{0}}^{t} G_{i j}(t, u) \mathrm{d} u, & \text { for } i \neq j
\end{array} \quad, \quad 1 \leq i, j \leq n,\right.
$$

and

$$
\begin{aligned}
G_{i j}\left(t, t_{0}\right)= & a_{i j}(t)+\sum_{k_{1}=1}^{n}\left(\int_{t_{0}}^{t} a_{i k_{1}}(u) \mathrm{d} u\right) a_{k_{1} j}\left(t_{0}\right) \\
& +\sum_{k=1}^{n} \sum_{k_{1}=1}^{n} \int_{t_{0}}^{t} a_{k_{1} k}(u) \mathrm{d} u \int_{t_{0}}^{t} a_{i k_{1}}(u) a_{k j}\left(t_{0}\right) \mathrm{d} u
\end{aligned}
$$

$$
1 \leq i, j \leq n
$$

truncating at the third composition power. The original publication addresses the limiting $n$th power, although with very unfortunate typographical mistakes throughout the text of which the reader must be aware.

For the simpler $2 \times 2$ system illustrated in Figure 5, the corresponding analytical solutions become,

$$
\begin{aligned}
M_{1}(t)= & M_{1}\left(t_{0}\right)\left(1+\int_{t_{0}}^{t} G_{11}(t, u) \mathrm{d} u\right)+M_{2}\left(t_{0}\right)\left(\int_{t_{0}}^{t} G_{12}(t, u) \mathrm{d} u\right) \\
& +\int_{t_{0}}^{t} f(u) \mathrm{d} u\left(1+\int_{t_{0}}^{t} G_{11}(t, u) \mathrm{d} u\right)+\int_{t_{0}}^{t} G_{12}(t, u) \mathrm{d} u,
\end{aligned}
$$


and

$$
\begin{aligned}
M_{2}(t)= & M_{1}\left(t_{0}\right) \int_{t_{0}}^{t} G_{21}(t, u) \mathrm{d} u+M_{2}\left(t_{0}\right)\left(1+\int_{t_{0}}^{t} G_{22}(t, u) \mathrm{d} u\right) \\
& +\int_{t_{0}}^{t} f(u) \mathrm{d} u \int_{t_{0}}^{t} G_{21}(t, u) \mathrm{d} u+1+\int_{t_{0}}^{t} G_{22}(t, u) \mathrm{d} u
\end{aligned}
$$

which get further simplified under the initial condition $M_{2}\left(t_{0}\right)=0$, as it usually happens in PK.

\section{Results and discussion}

Fractal PK, unlike an odd peculiarity, corresponds to the actual time course of drug molecules when found in heterogeneous, space-restricted, microenvironments. Thus, all drugs share this characteristic particularly in light of the biophase concept, earlier denoted as the 'effect site', and really meaning the immediate location where the drug interacts with the body, either in terms of a membrane interface, a metabolic enzyme or a pharmacological receptor. Although only a few handful of molecules elicit a self-similar systemic exponential decay, without ever reaching a true log-linear terminal phase, this is exactly the same behaviour that all drugs endure 'peripherally', often just clouded by too high lower limits of quantification. Essentially, only when the majority of drug molecules dwell in reasonably homogeneous well stirred media, such as the blood stream, the lymphatic system or even the interstitial fluid space, with only a fraction being partitioned into the deeper heterogeneous microenvironments, under a distribution steady-state, the classical compartmental homogeneous approximation works, either in terms of a small number of compartments or ultimately with just one. On the other hand, only more recently the bioanalytical advancements, both in instrumentation and control software, have brought the lower limits of quantification of drugs in biological matrices to new unprecedent lows. Before, sampling would just have to be cut short from revealing the full kinetic picture for analytical reasons. Now, drugs that have been consistently modelled over the years under the homogeneity assumption are in many cases disclosing their fractal kinetics characteristics when followed over time to lower and lower concentrations. Lastly, there are drug molecules that partition so significantly into all the recondite fractal environments in the body, that even with little effort their firstly coined 'strange kinetics' becomes evident. Such is the case of radioisotopes of so-called bone-seekers like calcium, strontium, barium and radium and their trace-kinetics, as identified in the very early physiology literature [52], and more recently corroborated in terms of non-radioactive calcium whole biodistribution [45]. Fitting those early data with a sum of exponential terms resulted in a different number of exponentials being needed depending on the time range chosen. Alternatively, the empirical equations then used for their smaller number of parameters, namely $y(t)=a t^{-\alpha}$ and $y(t)=a t^{-\alpha} \mathrm{e}^{-\beta t}$ [82], may now be recognized in Equations (27) and (28) above.

Figure 7 illustrates one of those cases for ${ }^{47} \mathrm{Ca}$ from long time ago [5], where the original authors used two power functions to describe plasma specific activities measured in rats. About tissue distribution they came to the conclusion that

equilibration is never in fact complete and kinetics depending on fixed compartments and exchange rates would not be a good description of this type of exchange. There is a barrier between the exchange of calcium and the blood, extracellular fluid and the tissues, which 

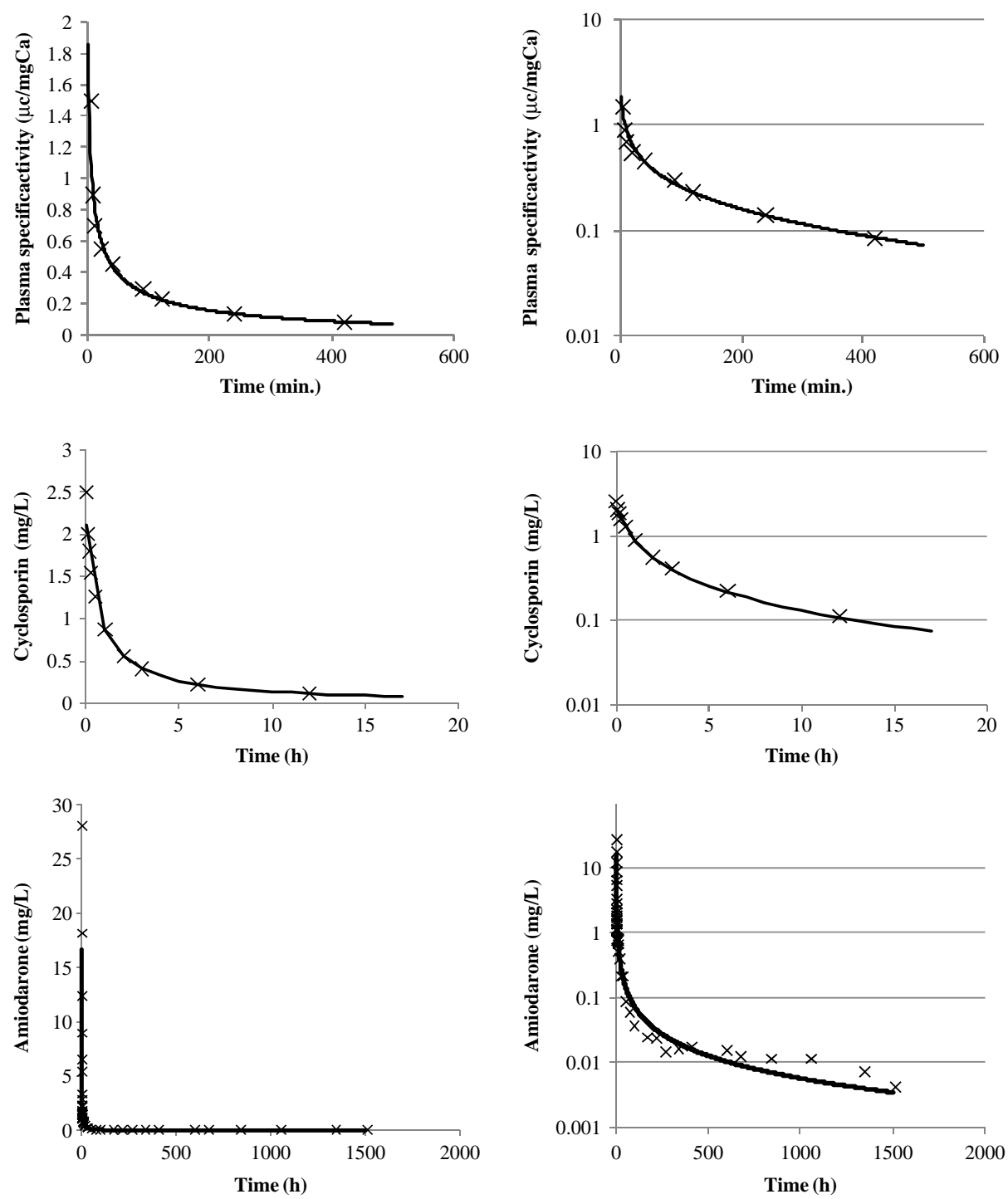

Figure 7. Examples of data published as having anomalous, strange or heterogeneous kinetics in different time scales, fitted with a two compartment fractal model as described in Figure 5: First row, ${ }^{47} \mathrm{Ca}$ data from Anderson and Tomlinson [5]; Second row, Cyclosporin data from Claret et al. [16]; Third row, Amiodarone data from Tucker et al. [76] as shown also in Figure 6. On the left column, data are plotted in linear scales while on the right, the same data are found in semilog plots.

appears to alter with time in various tissues. At present it is not possible to describe this mathematical function in a satisfactory manner (sic.).

Nowadays, it seems self-evident that these 'barriers' correspond to the fractal nature of the tissular microenvironments and the reason for a true distribution steady-state never to be reached is the conceptually infinite detail with which those tissues may be described relative to the diffusion of such a small molecule like calcium. Its like trying to soak a sponge that keeps finding more and more imbedded spaces available for water molecules 
to get into. On the comment about a satisfactory mathematical description of this reality, as it was hopefully illustrated above, there are now better insights on how to do it than at the time of the study.

Another example also depicted in Figure 7 is the more recent work about the heterogeneous PK of cyclosporin [16]. This is a drug with documented binding peculiarities, extensive and multiple metabolism, and deep tissue distribution characteristics. As these authors wrote, "the concept of "homogeneous compartment" was used in order to simplify the prototype system and make the mathematical analysis feasible. Later on, due to the complexity and the diversity of observed data, more complex multicompartmental models were developed' (sic.), having themselves suggested a power-law stochastic modelling approach. The fact of the matter is that, in this case, the whole disposition process begs for a fractal description. To capture all its intricacies either an endless number of compartments or a robust variance model are needed, with inherent applicability or interpretation issues. Alternatively, a fractal state-space approach, with a solid diffusion-based derivation, may provide not just descriptive power but also inference capability.

Finally, perhaps the most explicit example of a drug eliciting clear fractal PK is amiodarone, as firstly identified by Tucker et al. [76]. In Figure 7, again just one illustrative data set reveals now a degree of complexity even more challenging. The orders of magnitude covered by drug concentrations following a single dose administration resemble a series of matryoshkas always with one more chuckling inside. In this case, it is like squeezing the fractal 'sponge' endlessly and always continuing to see drug material coming out. Inevitably, the lower limit of quantification of the bioanalytical method used truncates this process when the heteroscedasticity of the data starts producing non-random residuals and regression anomalies. Amiodarone is known to take weeks to elicit maximal efficacy, since it distributes and stores in essentially all body tissues with very large saturation thresholds. Accordingly, it is not eliminated or excreted like most other drugs, but instead it seems to be leached out as amiodaronecontaining cells are lost from the body according to their own turnover. A pertinent implication of this fractal PK is the fact that amiodarone, being stored in so many different microenvironments, may produce adverse side effects in as many different organs and systems. It's both a class I and III antiarrhythmic agent, a beta-blocker, a calcium blocker, a vasodilator and a thyroid blocking agent, among other attributes. And most of these effects take so long to develop as they take to fade away upon drug discontinuation. In the process, drug may accumulate in the cornea causing halo-vision, in the skin causing blue-grey discolorations, in the thyroid causing hypo- or hyperthyroidism, in the liver causing hepatic disease, in the upper intestine causing severe gastric reflux, in the lungs causing acute lung syndrome and, on and on, as if the whole body was a macroscopic fractal network. It's most adequate PK modelling becomes thus undisputable.

Looking at the big picture, fractal PK ought to be considered as the general overall theory of which homogeneous compartmental analysis is just a part. As illustrated in Figure 8, according to Equation (35), when the fractal exponent $h$ is simulated to be close to zero, classical homogeneous kinetics results, identified for a two compartment model by the short-time upward curvature before a distribution steady-state is established, and by the clear log-linear terminal phase extrapolatable to infinity. When $h$ is simulated to approach unity, then sustained concentrations result as time increases, never reaching such log-linearity. 

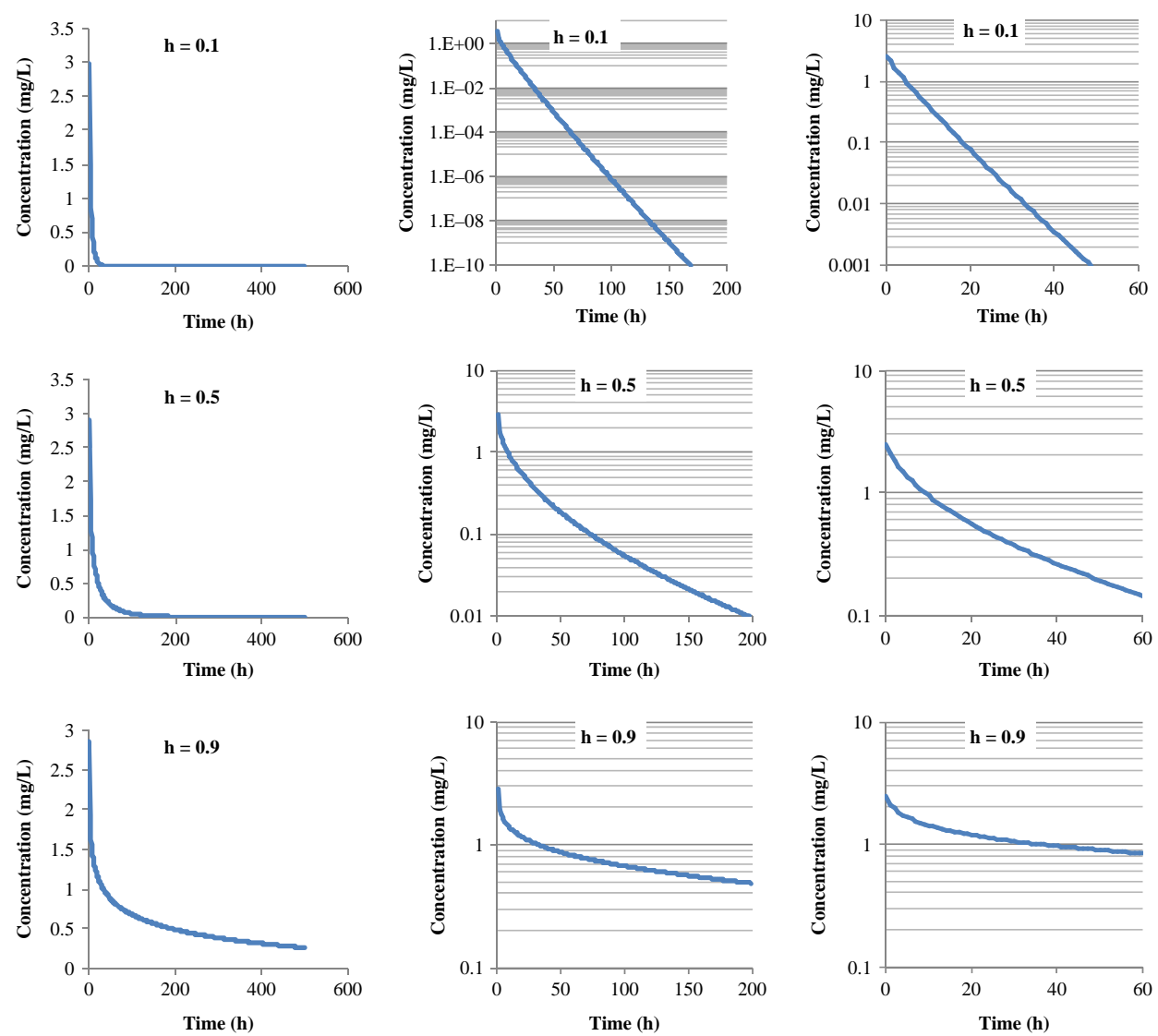

Figure 8. Simulations from the model and respective parameters described in Figure 6 changing the fractal exponent $h$ alone. Each row refers to the same data just plotted on a linear scale at the left, midway through on a semi-log scale on the middle and likewise just for a few early times on the right.

\section{Conclusions}

The early approach of fitting power functions with negative exponents to experimental data, although empirical, provided 'better long-term predictions of how much substance was left in the human body many weeks or months later. The corresponding estimates based on a polyexponential function were then out by $100 \%$ or more', in the words of Wise [82]. In the 1980s, he concluded that 'twenty-years later it is scarcely appreciated that all of this could apply, mutatis mutandis, to pharmacokinetic data'. Now, 40 years after the 1960s, one may dare to say nearly the same.

Nature, and therefore biological systems, is inherently fractal. As so many of its other aspects, this understanding escaped us for centuries, as many others will continue to escape for many more. Nevertheless, knowledge inexorably accrues over time regardless of mankind and it is just our choice to make use of it or not. As we stand today, it seems congruent that fractal PK encompasses all the required characteristics and explanations for all experimental observations related to the time course of drugs when administered to biological systems. Which means that it includes as sub-cases, the more common and previously exclusive compartmental, physiological and systems analyses. Therefore, it does not disprove them, nor does it replace them. It just provides the necessary insight for 
every time the underlying assumptions required for all those other modelling approaches are just not valid. The universal parsimony principle states it the best: 'pluralities ought not be supposed without necessity', William of Ockham (1285-1349). But as a contemporary of Smoluchowski put it more recently, 'We should make things as simple as possible, but not simpler' A. Einstein (1879-1955).

Every time the heterogeneity of living organisms, as opposed to physical ones, impacts on the whereabouts of drug molecules and thus on their kinetics, this will become fractal. Focusing on a diffusion rationale for the movement of each single molecule, a timedependent rate constant formalism may be conceived using fractal exponential parameters. This strategy may summarize the underlying phenomena under a small parameter space still amenable to be handled by standard statistics towards significance and inference issues. Fractal PK is reputable and it's available.

\section{References}

[1] S. Alexander and R. Orbach, Density of states on fractals: 'fractons', J. Phys. Lett. 43 (1982), pp. L625-L631.

[2] F. Amblard, A.C. Maggs, B. Yurke, A.N. Pargellis, and S. Leibler, Subdiffusion and anomalous viscoelasticity in actin networks, Phys. Rev. Lett. 77 (1996), pp. 4470-4473.

[3] L.W. Anacker and R. Kopelman, Fractal chemical kinetics: Simulations, and experiments, J. Chem. Phys. 81 (1984), pp. 6402-6403.

[4] L.W. Anacker, R. Kopelman, and J.S. Newhouse, Fractal chemical kinetics: Reacting random walkers, J. Stat. Phys. 36(5/6) (1984), pp. 591-602.

[5] J. Anderson and R.W.S. Tomlinson, The distribution of calcium-47 in the rat, J. Physiol. 182 (1966), pp. 664-670.

[6] J. Anderson, S.B. Osborn, R.W. Tomlinson, and M.A. Weinbren, Some applications of power law analysis to radioisotope studies in man, Phys. Med. Biol. 8(3) (1963), pp. 287-295.

[7] F.I. Ataullakhanov, S.V. Komarova, and V.M. Vitvitsky, A possible role of adenylate metabolism in human erythrocytes: simple mathematical model, J. Theor. Biol. 179 (1996), pp. 75-86.

[8] E. Barkai and J. Klafter, Comment on 'Subdiffusion and anomalous viscoelasticity in actin networks', Phys. Rev. Lett. 81 (1998), p. 1134.

[9] A.H. Beckett and G.T. Tucker, Problems in the in vivo evaluation of drug preparations and the interpretation of in vivo data, J. Mond. Pharm. 3 (1967), pp. 181-202.

[10] D. ben-Avraham and S. Havlin, Diffusion and Reactions in Fractals and Disordered Systems, Cambridge University Press, Cambridge, 2000.

[11] F. Brouers and O. Sotolongo-Costa, Generalized fractal kinetics in complex systems. Applications to biophysics and biotechnology, Phys. A: Stat. Mech. Appl. 368(1) (2006), pp. $165-175$.

[12] F. Brouers, O. Sotolongo-Costa, and K. Weron, Burr, Lévy, Tsallis, Phys. A: Stat. Mech. Appl. 344(3-4) (2004), p. 409.

[13] F.X. Caroli-Bosc, A. Iliadis, L. Salmon, P. Macheras, A.M. Montet, A. Bourgeon, R. Garrafo, J.P. Delmont, and J.C. Montet, Ursodeoxycholic acid modulates cyclosporin A oral absorption in liver transplant recipients, Fundam. Clin. Pharmacol. 14 (2000), pp. 601-609.

[14] P. Chelminiak, R. Marsh, J. Tuszynski, J.M. Dixon, and K.J.E. Vos, Asymptotic time dependence in the fractal pharmacokinetics of a two-compartment model, Phys. Rev. E 72(031903) (2005), pp. 1-7.

[15] B.S. Chen, Y.C. Wang, W.S. Wu, and W.H. Li, A new measure of the robustness of biochemical networks, Bioinformatics 21 (2005), pp. 2698-2705.

[16] L. Claret, A. Iliadis, and P. Macheras, A stochastic model describes the heterogeneous pharmacokinetics of cyclosporin, J. Pharmacokinet. Pharmacodyn. 28(5) (2001), pp. 445-463.

[17] E.A. Coddington and N. Levinson, Theory of Ordinary Differential Equations, McGraw-Hill, New York, 1955.

[18] R. Curto, E.O. Voit, A. Sorribas, and M. Cascante, A power-law model of purine metabolism: comparison of alternative modelling strategies, Math. Biosci. 151 (1998), pp. 1-49.

[19] Y. Dassas and P. Duby, Diffusion toward fractal interfaces, J. Electrochem. Soc. 142 (1995), p. 4175. 
[20] A. Dokoumetzidis and P. Macheras, A population growth model of dissolution, Pharm. Res. 14 (1997), pp. 1122-1126.

[21] A. Dokoumetzidis and P. Macheras, A tube model for transport and dispersion in the circulatory system based on the vascular fractal tree, Ann. Biomed. Eng. 31 (2003), pp. $284-293$.

[22] J. Fuite, R. Marsh, and J. Tuszynski, Fractal pharmacokinetics of the drug mibefradil in the liver, Phys. Rev. E 66(021904) (2002), pp. 1-11.

[23] R. Grima and S. Schnell, How reaction kinetics with time-dependent rate coefficients differs from generalized mass action, Chem. Phys. Chem. 7 (2006), pp. 1422-1424.

[24] R. Heinrich and S. Schuster, The Regulation of Cellular Systems, Chapman and Hall, New York, 1996.

[25] K. Higaki, S. Yamashita, and G.L. Amidon, Time-dependent oral absorption models, J. Pharmacokinet. Pharmacodyn. 28 (2001), pp. 109-128.

[26] T.H. Hughes and J.H. Matis, An irreversible two-compartment model with age-dependent turnover rates, Biometrics 40 (1984), pp. 501-505.

[27] B.D. Hughes, M.F. Schlesinger, and E.W. Montroll, Random walks in self similar clusters, Proc. Natl Acad. Sci. USA 78 (1981), pp. 3287-3291.

[28] V. Karalis and P. Macheras, Drug disposition viewed in terms of the fractal volume of distribution, Pharm. Res. 19 (2002), pp. 696-703.

[29] V. Karalis, A. Dokoumetzidis, and P. Macheras, A physiologically based approach for the estimation of recirculatory parameters, J. Pharmacol. Exp. Ther. 308 (2004), pp. 198-205.

[30] V. Karalis, A. Tsantili-Kakoulidou, and P. Macheras, Multivariate statistics of disposition pharmacokinetic parameters for structurally unrelated drugs used in therapeutics, Pharm. Res. 19 (2002), pp. $1827-1834$.

[31] V. Karalis, A. Tsantili-Kakoulidou, and P. Macheras, Quantitative structure pharmacokinetic relationships for disposition parameters of cephalosporins, Eur. J. Pharm. Sci. 20 (2003), pp. 115-123.

[32] V. Karalis, L. Claret, A. Iliadis, and P. Macheras, Fractal volume of drug distribution: it scales proportionally to body mass, Pharm. Res. 18 (2001), pp. 1056-1060.

[33] H.P. Koch and H. Zacek, Fractals also in pharmacokinetics, Pharmazie 46(H12) (1991), pp. $870-871$.

[34] R. Kopelman, Rate processes on fractals: theory, simulations, and experiments, J. Stat. Phys. 42(1/2) (1986), pp. 185-200.

[35] R. Kopelman, Fractal reaction kinetics, Science 241 (1988), pp. 1620-1626.

[36] K. Kosmidis, P. Argyrakis, and P. Macheras, Fractal kinetics in drug release from finite fractal matrices, J. Chem. Phys. 119 (2003), pp. 6373-6377.

[37] M. Kotulski and K. Weron, Random walk approach to relaxation in disordered systems, in Athens Conference on Applied Probability and Time Series Analysis Volume I: Applied Probability, C.C. Heyde, Y.V. Prohorov, R. Pyke, and S.T. Rachev, eds., Lecture Notes in Statistics 114, Springer, New York, 1996, pp. 379-388.

[38] P. Lansky and M. Weiss, Does the dose-solubility ratio affect the mean dissolution time of drugs?, Pharm. Res. 16 (1999), pp. 1470-1476.

[39] P. Lansky and M. Weiss, Modeling heterogeneity of properties and random effects in drug dissolution, Pharm. Res. 18 (2001), pp. 1061-1067.

[40] P. Lansky and M. Weiss, Classification of dissolution profiles in terms of fractional dissolution rate and a novel measure of heterogeneity, J. Pharm. Sci. 92 (2003), pp. 1632-1647.

[41] A. Le Mehaute, Transfer processes in fractal media, J. Stat. Phys. 36(5/6) (1984), pp. 665-676.

[42] A. Le Mehaute and G. Crepy, Introduction to transfer and motion in fractal media: The geometry of kinetics, Solid State Ionics 9(10) (1983), p. 17.

[43] X.-F. Li, Approximate solution of linear ordinary differential equations with variable coefficients, Math. Comput. Simulation 75(3-4) (2007), pp. 113-125.

[44] P. Macheras, Carrier-mediated transport can obey fractal kinetics, Pharm. Res. 12(4) (1995), pp. 541-548.

[45] P. Macheras, A fractal approach to heterogeneous drug distribution: calcium pharmacokinetics, Pharm. Res. 13 (1996), pp. 663-670.

[46] P. Macheras and A. Argyrakis, Gastrointestinal drug absorption: Is it time to consider heterogeneity as well as homogeneity, Pharm. Res. 14 (1997), pp. 842-847. 
[47] P. Macheras and A. Dokoumetzidis, On the heterogeneity of drug dissolution and release, Pharm. Res. 17 (2000), pp. 108-112.

[48] F. Mainardi, Fractional relaxation in anelastic solids, J. Alloys Comp. 211(212) (1994), pp. $534-538$.

[49] B.B. Mandelbrot, The Fractal Geometry of Nature, W.H. Freeman, San Francisco, CA, 1983.

[50] R. Marsh and J.A. Tuszynski, Fractal Michaelis-Menten kinetics under steady-state conditions: Application to mibefradil, Pharm. Res. 23(12) (2006), pp. 2760-2767.

[51] R.E. Marsh, T.A. Riauka, and S.A. McQuarrie, Use of a simulated annealing algorithm to fit compartmental models with an application to fractal pharmacokinetics, J. Pharm. Pharmaceut. Sci. 10(2) (2007), pp. 168-179.

[52] G.H. Marshall, Calcium pools and the power function, in Compartments, Pools and Spaces in Medical Physiology, P.E. Bergner and C.C. Lushbaugh, eds., Proceedings of AEC Symposium Series no.11, Oak Ridge, 1966, pp. 451-468.

[53] H.P. McKean, Hausdorff-Besicovitch dimension of Brownian motion paths, Duke Math. J. 22(2) (1955), pp. 229-234.

[54] K.S. Miller and B. Ross, An Introduction to the Fractional Calculus and Fractional Differential Equations, Wiley, New York, 1993.

[55] R.R. Nigmatullin, On the theory of relaxation with 'remnant' memory, Phys. Status Solidi B 124 (1984), pp. 389-393.

[56] R.R. Nigmatullin, The realization of the generalized transfer in a medium with fractal geometry, Phys. Status Solidi B 133 (1986), p. 425.

[57] W.P. Norris, S.A. Tyler, and A.M. Brues, Retention of radioactive bone-seekers: calculations based on the power function cast doubt on the present concept of biological half-times, Science 128(3322) (1958), pp. 456-462.

[58] T. Ogihasra, I. Tamai, and A. Tsuji, Application of fractal kinetics for carrier-mediated transport of drugs across intestinal epithelial membrane, Pharm. Res. 4 (1998), pp. 620-625.

[59] B. O'Shaughnessy and I.I. Procaccia, Analytical solution for diffusion on fractal objects, Phys. Rev. Lett. 54(5) (1985), pp. 455-458.

[60] L.M. Pereira, Comment on 'Asymptotic time dependence in the fractal pharmacokinetics of a two-compartment model', Phys. Rev. E 78 (2008), p. 5.

[61] A. Rescigno, The rise and fall of compartmental analysis, Pharmacol. Res. 44 (2001), pp. $337-342$.

[62] L.F. Richardson, Atmosphere diffusion shown on a distance-neighbour graph, Proc. R. Soc. Lond. A. 110 (1926), p. 709.

[63] J. Rudnik and G. Gaspari, The shapes of random walks, Science 237(4813) (1987), pp. $384-389$.

[64] A.I. Saichev and G.M. Zaslavsky, Fractional kinetic equations: Solutions and applications, Chaos 7(4) (1997), pp. 753-764.

[65] M.A. Savageau, Biochemical systems analysis: i. Some mathematical properties of the rate law for component enzymatic enzymatic reactions, J. Theor. Biol. 25 (1969), pp. 365-369.

[66] M.A. Savageau, Michaelis-Menten mechanism reconsidered: Implications of fractal kinetics, J. Theor. Biol. 176 (1995), pp. 115-124.

[67] M.A. Savageau, Development of fractal kinetic theory for enzyme-catalysed reactions and implications for the design of biochemical pathways, Biosystems 47 (1998), pp. 9-36.

[68] S. Schnell and T.E. Turner, Reaction kinetics in intracellular environments with macromolecular crowding: simulations and rate laws, Prog. Biophys. Mol. Biol. 85 (2004), pp. $235-260$.

[69] V. Seshadri and B.J. West, Fractal dimensionality of Lévy processes, Proc. Natl Acad. Sci. USA 79 (1982), pp. 4501-4505.

[70] M.V. Smoluchowski, Versuch einer mathematischen Theorie der Koagulationskinetik kolloider Lösungen, Z. Phys. Chem. 92 (1917), pp. 129-168.

[71] D. Stauffer, Introduction to Percolation Theory, Taylor \& Francis, London, 1985.

[72] T. Teorell, Kinetics of distribution of substances administered to the body, Arch. Intern. Pharmacodyn. Ther. 57 (1937), pp. 205-225.

[73] C. Tsallis, Nonextensive statistical mechanics and thermodynamics. Historical background and present status, in Nonextensive Statistical Mechanics and its Applications, S. Abe and Y. Okamoto, eds., Series Lecture Notes in Physics, Springer, Berlin, 2001, pp. 3-98. 
[74] C. Tsallis, Dynamical scenario for nonextensive statistical mechanics, Phys. A: Stat. Mech. Appl. 340(1-3) (2004), pp. 1-10.

[75] G.T. Tucker, Compartmental vs. empirical vs. physiological models, in Topics in Pharmaceutical Sciences, D.D. Breimer and P. Speiser, eds., Elsevier, Amsterdam, 1982, pp. 33-48.

[76] G.T. Tucker, P.R. Jackson, and G.C.A. Storey, Amiodarone disposition: poliexponential, power and gamma functions, Eur. J. Clin. Pharmacol. 26 (1984), pp. 655-656.

[77] G. Valsami, A. Dokoumetzidis, and P. Macheras, Modeling of supersaturated dissolution data, Int. J. Pharm. 181 (1999), pp. 153-157.

[78] S. Vasilach, Algebraic method for solving systems of linear differential equations with variable coefficients, SIAM J. Math. Anal. 12(1) (1981), pp. 23-39.

[79] M. Weiss, The anomalous pharmacokinetics of amiodarone explained by nonexponential tissue trapping, J. Pharmacokinet. Biopharm. 27 (1999), pp. 383-396.

[80] M.E. Wise, The evidence against compartments, Biometrics 27 (1971), p. 262.

[81] M.E. Wise, Interpreting both short- and long-term power laws in physiological clearance curves, Math. Biosci. 20 (1974), pp. 327-337.

[82] M.E. Wise, Negative power functions of time in pharmacokinetics and their implications, J. Pharmacok. Pharmacodyn. 13(3) (1985), pp. 309-346.

[83] M.E. Wise and J.M. Borsboom, Two exceptional sets of physiological clearance curves and their mathematical form: Test cases?, Bull. Math. Biol. 51 (1989), pp. 579-596.

[84] M. Yamamoto, On the homogeneous linear systems of differential equations with variable coefficients, Proc. Japan Acad. Ser. A 53(1) (1977), pp. 17-20.

[85] G.M. Zaslavsky, Chaos, fractional kinetics, and anomalous transport, Phys. Rep. 371 (2002), pp. $461-580$. 


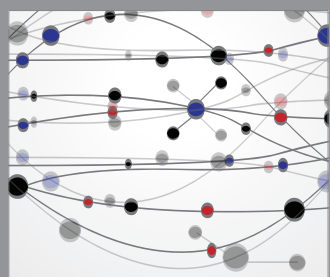

The Scientific World Journal
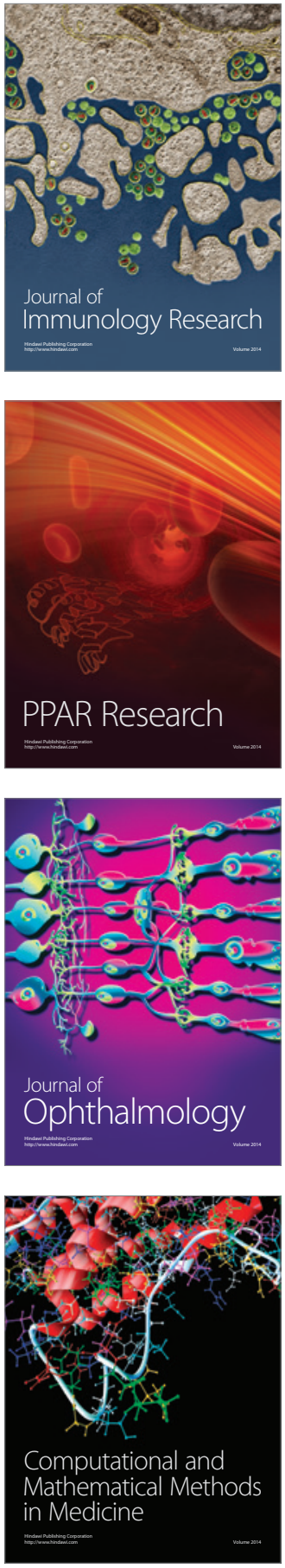

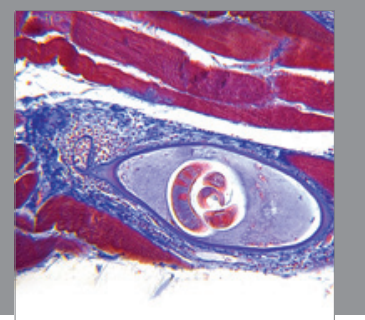

Gastroenterology

Research and Practice
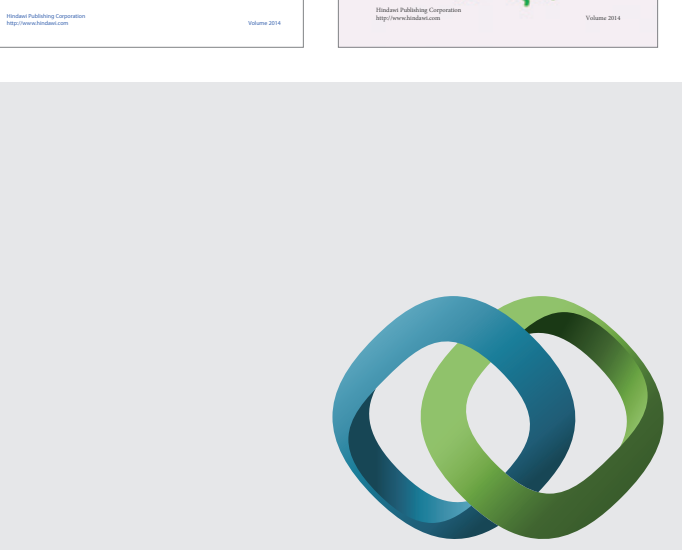

\section{Hindawi}

Submit your manuscripts at

http://www.hindawi.com
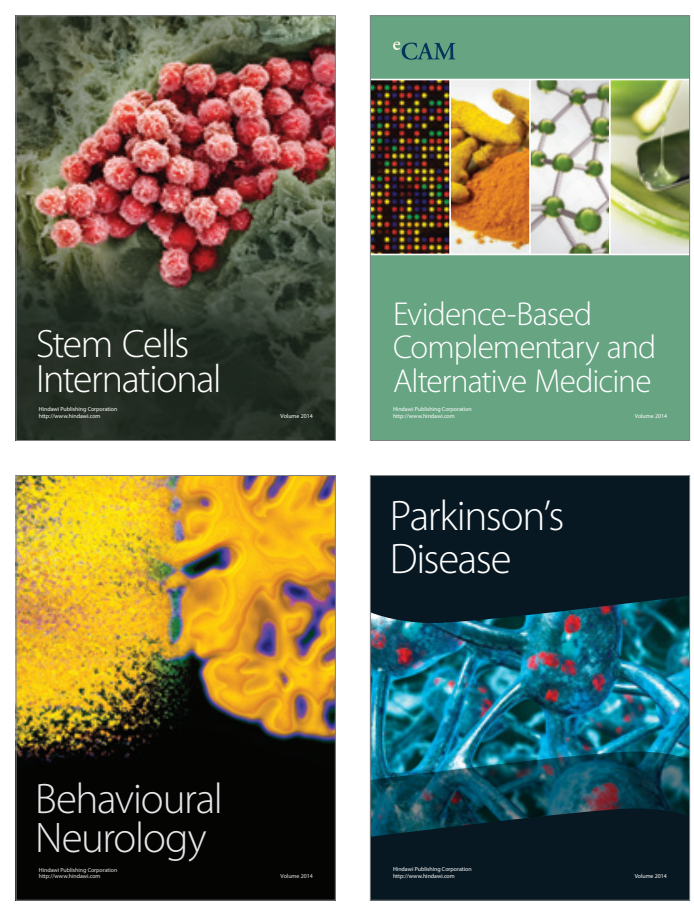

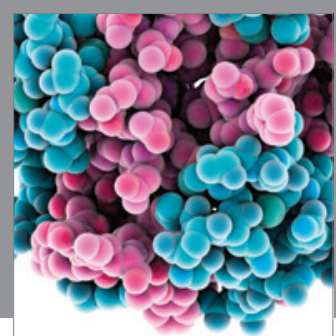

Journal of
Diabetes Research

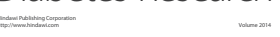

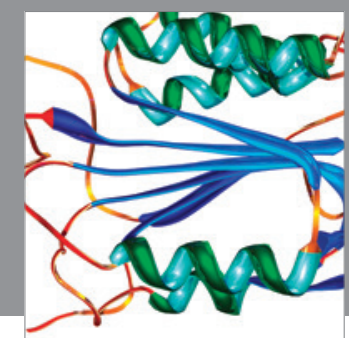

Disease Markers
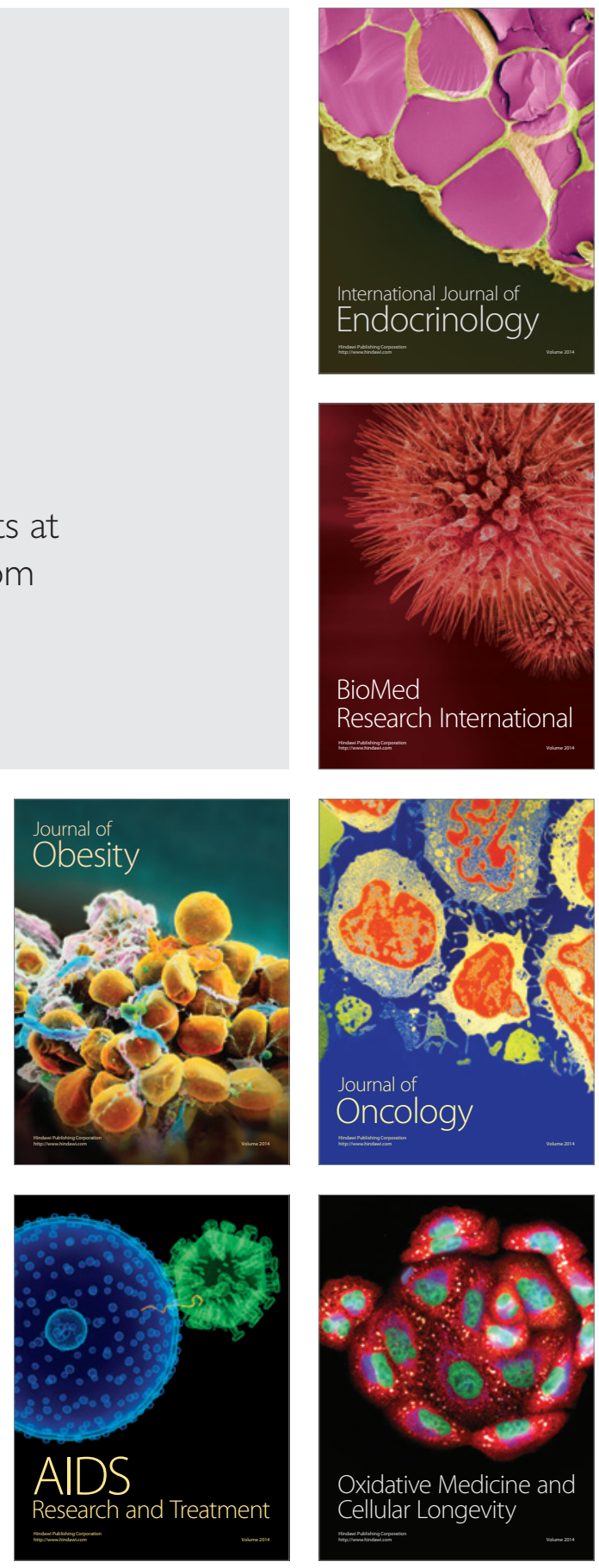